\title{
Bright-dark rogue wave in mode- locked fibre laser (Conference Presentation)
}

Hani Kbashi

Stanislav Kolpakov

Amós Martinez

Chengbo Mou

Sergey V. Sergeyev 


\title{
Bright-dark rogue wave in mode-locked fibre laser (Conference Presentation)
}

\author{
Hani Kbashi, Aston Univ. (United Kingdom); Stanislav A. Kolpakov, Amós Martinez, Aston \\ Institute for Photonics Technologies, Aston Univ. (United Kingdom); Chengbo Mou, Shanghai \\ Univ. (China); Sergey V. Sergeyev, Aston Institute for Photonics Technologies, Aston Univ. \\ (United Kingdom)
}

\author{
ABSTRACT \\ Bright-Dark Rogue Wave in Mode-Locked Fibre Laser \\ Hani Kbashi1*, Amos Martinez1, S. A. Kolpakov1, Chengbo Mou, Alex Rozhin1, Sergey V. Sergeyev1 \\ 1Aston Institute of Photonic Technologies, School of Engineering \& Applied Science Aston University, \\ Birmingham, B4 7ET, UK \\ kbashihj@aston.ac.uk, 00447553534388
}

Keywords: Optical rogue wave, Bright-Dark rogue wave, rogue wave, mode-locked fiber laser, polarization instability.

Abstract:

Rogue waves (RWs) are statistically rare localized waves with high amplitude that suddenly appear and disappear in oceans, water tanks, and optical systems [1]. The investigation of these events in optics, optical rogue waves, is of interest for both fundamental research and applied science. Recently, we have shown that the adjustment of the incavity birefringence and pump polarization leads to emerge optical RW events [2-4]. Here, we report the first experimental observation of vector bright-dark RWs in an erbium-doped stretched pulse mode-locked fiber laser. The change of induced in-cavity birefringence provides an opportunity to observe RW events at pump power is a little higher than the lasing threshold. Polarization instabilities in the laser cavity result in the coupling between two orthogonal linearly polarized components leading to the emergence of bright-dark RWs. The observed clusters belongs to the class of slow optical RWs because their lifetime is of order of a thousand of laser cavity roundtrip periods.

References:

1. D. R. Solli, C. Ropers, P. Koonath,and B. Jalali, Optical rogue waves," Nature, 450, 1054-1057, 2007.

2. S. V. Sergeyev, S. A. Kolpakov, C. Mou, G. Jacobsen, S. Popov, and V. Kalashnikov, "Slow deterministic vector rogue waves," Proc. SPIE 9732, 97320K (2016).

3. S. A. Kolpakov, H. Kbashi, and S. V. Sergeyev, "Dynamics of vector rogue waves in a fiber laser with a ring cavity," Optica, 3, 8, 870, (2016).

5. S. Kolpakov, H. Kbashi, and S. Sergeyev, "Slow optical rogue waves in a unidirectional fiber laser," in

Conference on Lasers and Electro-Optics, OSA Technical Digest (online) (Optical Society of America, 2016), paper JW2A.56.

View presentation recording on the SPIE Digital Library:

http://dx.doi.org/10.1117/12.2265038.5463341892001

Nonlinear Optics and Applications X, edited by Mario Bertolotti, Joseph W. Haus, Alexei M. Zheltikov, Proc. of SPIE Vol. 10228, 102280P · () 2017 SPIE · CCC code: 0277-786X/17/\$18 · doi: 10.1117/12.2265038 